Trauma Berufskrankh 2018 20 (Suppl 1):S6-S8 DOI 10.1007/s10039-017-0268-4

Online publiziert: 20. Juni 2017

c) Springer Medizin Verlag Berlin 2017

CrossMark

\section{Schneidmueller ${ }^{1}$ C. von Rüden ${ }^{1,2} \cdot$ V. Bühren ${ }^{1}$}

'Abteilung für Unfallchirurgie, Sportorthopädie und Kindertraumatologie in Kooperation mit der BG Unfallklinik Murnau, Klinikum Garmisch-Partenkirchen, Murnau, Deutschland

${ }^{2}$ Universitätsinstitut für Biomechanik, Paracelsus Medizinische Privatuniversität, Salzburg, Österreich

\title{
Alternative Operationstechniken zur Behandlung von Femurschaftfrakturen bei Kindern und Jugendlichen
}

Mit zunehmender Mobilität steht die operative Versorgung der Femurschaftfraktur im Kindes- und Jugendalter im Vordergrund. Im deutschsprachigen Raum hat man sich auf eine Altersgrenze von etwa 3 Jahren geeinigt. Seit Einführung der elastisch stabilen intramedullären Nagelung (ESIN) in den 1980erJahren gilt diese als Goldstandard zur Stabilisierung von Femurschaftfrakturen im Kindesalter. Am besten geeignet sind kurze Querfrakturen der Schaftmitte, da hier die ESIN ihr biomechanisches Prinzip der 3-Punkt-Abstützung am besten realisieren kann. Damit lässt sich bei diesen längsstabilen Frakturen eine Übungsstabilität, meist sogar eine Belastungsstabilität erreichen [3].

In der Literatur finden sich viele Studien, die über gute Resultate der ESIN berichten. Viele dieser Untersuchungen beinhalten hinsichtlich der Femurfraktur jedoch keine adoleszenten Kinder oder machen keine Angaben über das Körpergewicht der Heranwachsenden [2, 8]. Garner et al. [4] führten eine Matchedpair-Analyse bezüglich des Körpergewichts durch und konnten im Vergleich von ESIN und konventioneller Marknagelosteosynthese keinen signifikanten Unterschied beobachten. Damit sahen sie keine Indikationsbeschränkung für die ESIN bei älteren bzw. schwereren Kindern.

Auf der anderen Seite gibt es eine Reihe von Untersuchungen, die abhängig von Alter und Gewicht eine zunehmen- de Komplikationsrate beobachteten. Sagan et al. [12] fanden ab einem Gewicht von 46,5 kg signifikant häufiger Achsabweichungen als bei leichteren Kindern. Sink et al. [13] konnte durch Halbierung der ESIN-Anwendung die Komplikationsrate von $52 \%$ auf $28 \%$ senken, mit einer Altersbeschränkung von 12 Jahren von $76 \%$ auf $12 \%$ und einer Gewichtsbegrenzung von $50 \mathrm{~kg}$ von $71 \%$ auf $15 \%$. In einer biomechanischen Untersuchung am Modell beobachteten Li et al. [9] ab einem Gewicht von 40-45 kg zunehmende Achsabweichungen. Ebenso fanden Moroz et al. [11] in einer Multicenterstudie gute Ergebnisse für ein Körpergewicht von $39 \mathrm{~kg}$, dagegen ein schlechtes Outcome bei einem Gewicht von $54 \mathrm{~kg}$. Die Richtlinien der American Academy
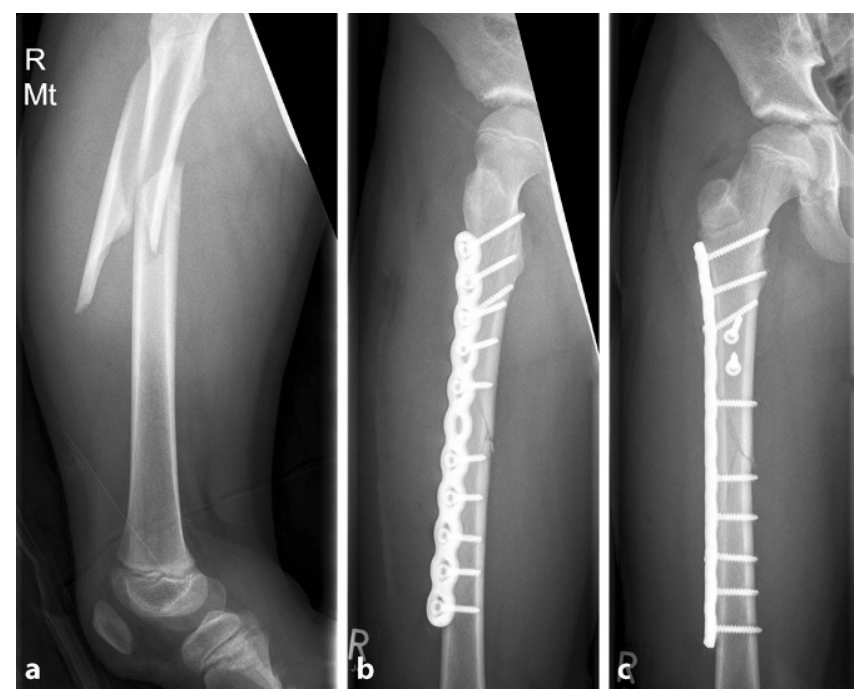

Abb. $1<10$-jähriger Junge nach Hochrasanztrauma mit einer mehrfragmentären proximalen Femurschaftfraktur und Plattenosteosynthese. a Unfallbild; b, c postoperatives Ergebnis. (Aus [14]) 
Aber gerade bei Jugendlichen gleichen die Konsolidierungszeiten denen bei Erwachsenen, sodass neben Komfortgründen ein Risiko in sog. PintrackInfektionen bei relativ langen Tragezeiten gesehen wird. Miner und Carroll [10] erfassten in ihrem Kollektiv eine durchschnittliche Tragedauer des Fixateurs von 107 Tagen mit einer antibiotisch behandlungspflichtigen Pintrack-Infektionsrate von etwa $73 \%$. Zudem wurde bei $22 \%$ der Kinder nach Fixateurentfernung eine Refraktur beobachtet.

Somit dient der Fixateur externe meist zur temporären Stabilisierung bis zum sekundären Verfahrenswechsel.

\section{Plattenosteosynthese}

Die Plattenosteosynthese ermöglicht ebenso eine Stabilisierung von längsinstabilen Frakturtypen und gewinnt in den letzten Jahren wieder zunehmend an Bedeutung [5, 13]. Gerade bei den proximalen oder distalen Femurfrakturen, die sich schlecht durch intramedulläre Verfahren stabilisieren lassen, stellt sie eine gute Alternative dar (• Abb. 1). Da im Schaftbereich keine anatomische Stellung erreicht werden muss, genügen eine achsgerechte Reposition und ein minimalinvasives Vorgehen mittels „eingeschobener" winkelstabiler Plattenosteosynthese (minimalinvasive Plattenosteosynthese [MIPO]).

\section{Marknagel}

Der konventionelle antegrade Marknagel stellt eine Alternative bei Jugendlichen mit bereits verschlossenen oder im Verschluss befindlichen Wachstumsfugen dar (-Abb. 2). Problematisch kann der meist sehr enge Markraum im Jugendalter sein, sodass ein herkömmlicher Nagel aus der Erwachsenentraumatologie ggf. nicht passt. Zum anderen besteht durch Verletzung der A. circumflexa am Eintrittspunkt die Gefahr der Durchblutungsstörung mit nachfolgender Wachstumsstörung des Schenkelhalses oder der Entwicklung einer Femurkopfnekrose [1]. Aus diesen Gründen wurde vor einigen Jahren ein lateraler Femurmarknagel speziell für Adoleszente entwickelt. Dieser ist in geringeren Durchmessern

Trauma Berufskrankh 2018 20 (Suppl 1):S6-S8 DOI 10.1007/s10039-017-0268-4

(c) Springer Medizin Verlag Berlin 2017

\section{Schneidmueller · C. von Rüden · V. Bühren}

\section{Alternative Operationstechniken zur Behandlung von Femurschaftfrakturen bei Kindern und Jugendlichen}

\section{Zusammenfassung}

Mit zunehmendem Mobilitätsbedürfnis steht die operative Therapie der Femurschaftfraktur bei Kindern und Jugendlichen im Vordergrund. Seit Einführung der elastisch stabilen intramedullären Nagelung (ESIN) in den 1980er-Jahren stellt sie den Goldstandard für die Osteosynthese dieser Frakturen dar. Mit immer größerer Ausweitung der Indikation häufen sich jedoch Berichte über Komplikationen dieser Technik. Neuere Studien legen nahe, dass gerade beim älteren und schwereren Kind die ESIN an ihre biomechanischen Grenzen gelangt. Insbesondere in dieser Patientengruppe, aber auch bei allen anderen Frakturtypen, bei denen die ESIN keine sichere Stabilisierung gewährleisten kann, werden alternative Techniken empfohlen. Die Indikation des Fixateur externe liegt im Wesentlichen in der zügigen Stabilisierung bei Polytraumatisierung oder Anwendung bei schlechten Weichteilverhältnissen. Die Plattenosteosynthese stellt eine gute Alternative zur internen Fixierung proximaler oder distaler Femurfrakturen dar, kann jedoch auch - meist in der MIPO (minimalinvasive Plattenosteosynthese)Technik - für längsinstabile Frakturen des Schaftes verwendet werden. Der konventionelle antegrade Marknagel ist zum einen häufig nicht für den schmalen Markraum bei Adoleszenten geeignet, zum anderen birgt er die Gefahr einer Durchblutungsstörung aufgrund des Eintrittspunktes. Der speziell für das Kindesalter entwickelte laterale Adoleszentennagel ist in geringeren Durchmessern erhältlich und hat wegen seines lateralen Eintrittspunktes nicht das Risiko der Durchblutungsstörung. Letztendlich ist die Therapie der Femurschaftfraktur im Wesentlichen abhängig von Alter und Gewicht der Kinder. Um eine adäquate Osteosynthese für alle Frakturtypen, Gewichts- und Altersgruppen zu erzielen, müssen daher sämtliche Alternativen neben der ESIN beherrscht werden.

Schlüsselwörter

Adipositas · Platte - Nagel - Osteosynthese . Mobilität

\section{Alternative surgical techniques for the treatment of femoral shaft fractures in children and adolescents}

\section{Abstract}

Surgical therapy is the treatment of choice in the treatment of femoral shaft fractures in children and adolescents due to an increased need for mobility. Since elastic stable intramedullary nailing (ESIN) was introduced in the 1980s it has become the gold standard fixation technique for these fractures. Nevertheless, with broadening indications, more complications have been described due to this technique. Recent studies reported biomechanical limitations especially in older and in heavier children. In this group of patients but also all fracture types in which ESIN does not guarantee secure stabilization, alternative surgical techniques are recommended. Indications for the usage of external fixators for rapid fixation are polytrauma and poor soft tissue conditions. Plate fixation represents a good alternative to internal fixation of proximal or distal femoral fractures. As it is not necessary to achieve anatomic reduction, minimally invasive plate osteosynthesis (MIPO) is preferred. Conventional anterograde intramedullary nailing is often not suitable for the relatively small medullary cavity in adolescents. Another problem is the risk of impaired blood circulation depending on the entry point of the nail. The specially designed so-called adolescent lateral femoral nails are commercially available in smaller diameters. Due to its lateral entry point the risk of impaired blood flow is decreased. The choice of surgical therapy for femoral shaft fractures depends mainly on the young patient age and weight; therefore, different implant alternatives other than the ESIN technique for fracture stabilization may be selected.

Keywords

Obesity $\cdot$ Plate $\cdot$ Nail $\cdot$ Osteosynthesis $\cdot$ Mobility 

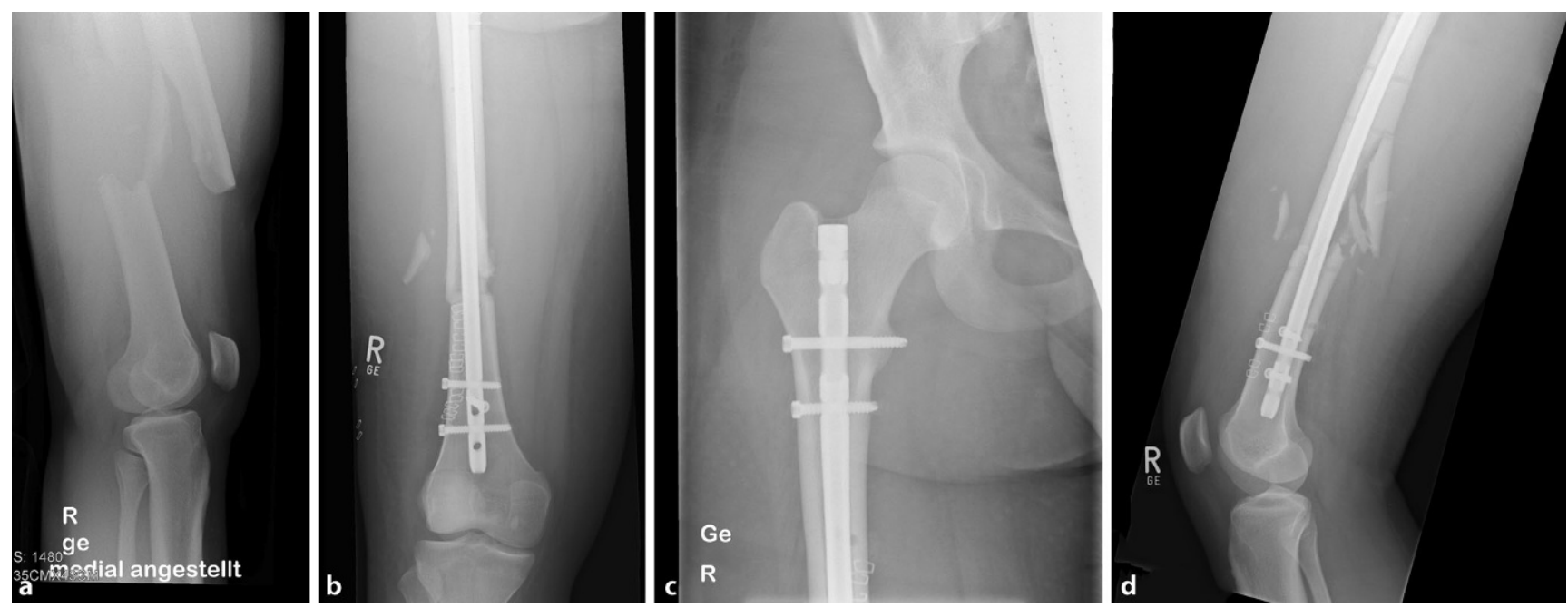

Abb. 2 ॥ Intramedullärer antegrader Verriegelungsnagel bei einem 14-jährigen Mädchen mit bereits verschlossenen Wachstumsfugen. a Unfallbild; b, c, d postoperatives Ergebnis. (Aus [14])

erhältlich und birgt nicht die Gefahr der Durchblutungsstörung durch einen lateralen Zugang über dem Trochanter major. Die Verwendung dieses Eintrittspunktes wird jedoch wegen der Gefahr der Wachstumshemmung der Apophyse des Trochanter major mit konsekutiver Valgisierung der Schenkelhalsachse nicht bei Kindern unter dem 8. Lebensjahr empfohlen. Manche Autoren geben sogar ein Mindestalter von 13 Lebensjahren an. Keeler et al. [6] beobachteten bei 80 mit Adolescent Lateral Femoral Nail (ALFN) versorgten Femurfrakturen und einem Durchschnittsalter von 12,9 (8 bis 18) Jahren keine Kopfnekrose oder Valgisierung des Schenkelhalses.

\section{Fazit für die Praxis}

- Die operative Therapie der Femurschaftfraktur steht beim mobilen lauffähigen Kind im Vordergrund.

- Der überwiegende Anteil lässt sich gut minimalinvasiv mittels ESIN stabilisieren.

- Gerade bei größeren und schwereren Kindern und Jugendlichen sollten jedoch alternative Techniken wie der Fixateur externe, die Platten- oder Marknagelosteosynthese beherrscht werden, um gute klinische Langzeitergebnisse zu gewährleisten.

\section{Korrespondenzadresse}

\section{PD Dr. D. Schneidmueller}

Abteilung für Unfallchirurgie, Sportorthopädie und Kindertraumatologie in Kooperation mit der BG Unfallklinik Murnau, Klinikum GarmischPartenkirchen

Professor-Küntscher Str. 8, 82418 Murnau,

Deutschland

dorien.schneidmueller@bgu-murnau.de

\section{Einhaltung ethischer Richtlinien}

Interessenkonflikt. D. Schneidmueller, C. von Rüden und V. Bühren geben an, dass kein Interessenkonflikt besteht.

Dieser Beitrag beinhaltet keine von den Autoren durchgeführten Studien an Menschen oder Tieren.

The supplement containing this article is not sponsored by industry.

\section{Literatur}

1. Buford D Jr, Christensen K, Weatherall P (1998) Intramedullary nailing of femoral fractures in adolescents. Clin Orthop Relat Res 350:85-89

2. Carey TP, Galpin RD (1996) Flexible intramedullary nail fixation of pediatric femoral fractures. Clin Orthop Relat Res 332:110-118

3. Dietz HG, Schmittenbecher PP, Slongo T, Wilkins E (Hrsg) (2006) Elastic stable intramedullary nailing (ESIN) in children. Thieme, Stuttgart New York

4. Garner MR, Bhat SB, Khujanazarov I et al (2011) Fixation of length-stable femoral shaft fractures in heavier children: flexible nails vs ridig locked nails. J Pediatr Orthop 31:11-16

5. Heyworth BE, Suppan CA, Kramer DE et al (2012) Management of pediatric diaphyseal femur fractures. Curr Rev Musculoskelet Med 5:120-125
6. Keeler KA, Dart B, Luhmann SJ (2009) Antegrad intramdeullary nailing of pediatric femoral fractures using an interlocking pediatric femoral nail and lateral trochanteric entry point. J Pediatr Orthop 29:245-351

7. KocherMS, SinkEL, Blasier RD et al (2010) American Academy ofOrthopaedic Surgeons clinical practice guideline on treatment of pediatric diaphyseal femur fracture. JBone Joint Surg Am 92:1790-1792

8. Lascombes P, Haumont T, Journeau P (2006) Use and abuse of flexible intramedullary nailing in children and adolescents. J Pediatr Orthop 26:827-834

9. Li YSK, Shilt JS (2008) Biomechanical analysis of titanium elastic nail fixation in pediatric femur fracture model. JPediatr Orthop 28:874-878

10. Miner T, Carroll KL (2000) Outcomes of external fixation of pediatric femoral shaft fractures. JPediatr Orthop 20:405-410

11. MorozLA, LaunayF, KocherMSetal (2006) Titanium elastic nailing of fractures oft he femur in children. Predictors of complications and poor outcome. JBone Joint Surg Br 88:1361-1366

12. Sagan ML, Datta JC, Olney BW et al (2010) Residual deformity after treatment of pediatric femur fractures with flexible titanium nails. J Pediatr Orthop 30:638-643

13. Sink EL, Faro F, Polousky J et al (2010) Decreased complications of pediatric femur fractures with a change in management. J Pediatr Orthop 30:633-637

14. Schneidmueller D, Kraft C, Bühren V et al (2014) Wachstumsverhalten nach Oberschenkelschaftfrakturen. Unfallchirurg 117(12):1099-1104. doi:10.1007/s00113-014-2634-z 